\title{
ESTADO NUTRICIONAL E DESENVOLVIMENTO MOTOR GROSSO DE LACTENTES ENTRE SEIS E DEZOITO MESES DE IDADE
}

\section{NUTRITIONAL STATUS AND GROSS MOTOR DEVELOPMENT OF INFANTS BETWEEN SIX AND EIGHTEEN MONTHS OF AGE}

\author{
Jaqueline da Silva Frônio ${ }^{1}$ \\ Alessandra Regina Coelho ${ }^{2}$ \\ Lillian Aparecida Graças ${ }^{3}$ \\ Luiz Cláudio Ribeiro ${ }^{4}$
}

Frônio JS et al. Estado nutricional e desenvolvimento motor grosso de lactentes entre seis e dezoito meses de idade. Rev. Bras. Cresc. e Desenv. Hum. 2011; 21(1): 30-38

\section{Resumo:}

Objetivo: verificar o desempenho motor grosso de lactentes de 6 a 18 meses de idade, por meio da Alberta Infant Motor Scale (AIMS), e se há associação entre este e o estado nutricional. Método: foi realizado estudo transversal, caso-controle, no qual foi avaliado o desenvolvimento motor grosso de lactentes entre 6 e 18 meses de idade por meio da AIMS, divididos em grupo de estudo (com desnutrição) e controle (sem desnutrição). Foram alocados 13 lactentes no grupo de estudo e 13 lactentes no grupo controle pareados quanto à idade, sexo e nível socioeconômico (cada grupo com 6 participantes do sexo feminino e 7 do masculino, e média de idade de 14,54 meses). Para comparação entre os grupos foram empregados os testes de Mann-Whitney (variáveis contínuas) e Exato de Fisher (variável categórica), sendo considerado um nível de significância de 0.05. Resultados: considerando tanto o Escore Bruto quanto o Índice percentil da AIMS, foi encontrada diferença estatisticamente significativa entre os grupos ( $p=0,023$ e $p=0,002$, respectivamente), tendo sido observado desempenho alterado (abaixo do percentil 5 da curva normativa da AIMS) em 10 (76,9\%) lactentes do grupo de estudo e em 2 (15,4\%) do grupo controle. Conclusão: os resultados indicam que a desnutrição parece estar negativamente associada ao desenvolvimento motor grosso de lactentes de 6 a 18 meses de idade, o que reafirma a importância de uma abordagem integral ao desnutrido que inclua a vigilância do desenvolvimento motor e, quando necessário, a realização de medidas que visem melhorar essa condição.

Palavras-chave: desenvolvimento infantil; lactente; desnutrição.

1 Doutora em Ciências Médicas pela Universidade Estadual de Campinas (UNICAMP), Professora Adjunta da Faculdade de Fisioterapia da Universidade Federal de Juiz de Fora (UFJF), Juiz de Fora, MG, Brasil.

Endereço: Universidade Federal de Juiz de Fora - Faculdade de Fisioterapia- Centro de Ciências da Saúde - R. José Lourenço Kelmer, s/n Campus Universitário- Bairro São Pedro - CEP: 36.036-900 Juiz de Fora- MG - E.mail: jaqueline.fronio@ufjf.edu.br

2 Fisioterapeuta graduada pela UFJF, Juiz de Fora, MG, Brasil. Rua Poeta Daltemar Lima, 120/201 - Bairro Encosta do Sol Juiz de Fora - MG CEP: 36083-015 - E. mail: lelecoelho1@yahoo.com.br

3 Fisioterapeuta graduada pela UFJF, Juiz de Fora, MG, Brasil. Rua João de Oliveira, 20 - Bairro São Francisco de Paula Juiz de Fora - MG CEP: 36087-490 - E.mail: lillifisio2005@yahoo.com.br

4 Doutor em Demografia pela UFMG, Professor Associado do Departamento de Estatística do Instituto de Ciências Exatas da UFJF, Juiz de Fora, MG, Brasil. - E.mail: luiz.claudio@ufjf.edu.br

Universidade Federal de Juiz de Fora - Departamento de Estatística- Instituto de Exatas - R. José Lourenço Kelmer, s/n Campus Universitário- Bairro São Pedro - CEP: 36.036-900 - Juiz de Fora- MG

O Estudo foi realizado pelo Núcleo Interdisciplinar de Estudos do Desenvolvimento Infantil (NIEDI) - Universidade Federal de Juiz de Fora (UFJF).

Correspondência para: Profa. Dra. Jaqueline da Silva Frônio - Universidade Federal de Juiz de Fora - Faculdade de FisioterapiaCentro de Ciências da Saúde - R. José Lourenço Kelmer, s/n Campus Universitário- Bairro São Pedro - CEP: 36.036-900 Juiz de Fora - MG - Telefones: (32) 2102-3843/4009-5318 - E-mail: jaqueline.fronio@ufjf.edu.br Projeto de Pesquisa financiado pela PROPESQ/UFJF através do programa BIC. 


\begin{abstract}
:
Objectives: to evaluate the gross motor performance in infants aged six to 18 months old, through the Alberta Infant Motor Scale (AIMS), and to verify if there is an association between the nutritional status and the gross motor performance. Methods: a cross-sectional case-control study which assessed the gross motor development of infants between six and 18 months old through the AIMS was conducted. Infants were divided into two study groups, the malnoutrished infants and the control group. Each group had 13 infants each, with 6 female participants and 7 male participants, equivalent in age, gender and socioeconomic status. The average age was 14,54 months old. The Mann-Whitney test (continuous variables) and the Exact Fisher Test (categorical variable) was used to compare both groups with a significance level of á=0.05. Results: considering the Raw score and the AIMS' centile index $(p=0,023$ e $p=0,002$, respectively) a statistically significant difference was found between the groups, observing poor performance (below the 5th centile of the AIMS' normative curve) in 10 (83,3\%) infants from the study group and in $2(16,7 \%)$ infants from the control group. Conclusion: the results indicate that malnutrition is negatively associated with gross motor development of infants aged 6 to 18 months old. This reaffirms the importance of an integrated approach to help malnourished infants, including the monitoring of motor development and, if necessary, implementation of measures to improve this condition.
\end{abstract}

Key words: child development; infant; malnutrition.

\section{INTRODUÇÃO}

Apesar das sucessivas conferências reafirmando o direito de todo ser humano não padecer de fome, a desnutrição infantil se mantém como um importante problema de saúde pública, principalmente nos países subdesenvolvidos e em desenvolvimento ${ }^{1}$.

A estimativa da Organização Mundial de Saúde (OMS) é que mais de 20 milhões (3,6\%) de crianças nascem com baixo peso a cada ano, aproximadamente 150 milhões (26,8\%) de crianças menores de cinco anos têm baixo peso para sua idade e 182 milhões (32,5\%) têm baixa estatura ${ }^{2}$. No Brasil, segundo dados da Pesquisa de Orçamentos Familiares - POF, 20022003, 4,6\% das crianças com menos de cinco anos são desnutridas (déficit de peso por idade) $)^{3}$.

Entre os principais fatores que contribuem para a desnutrição no lactente e na criança pequena encontram-se as suas necessidades proporcionalmente maiores de ingestão em relação aos demais membros da família, tanto de calorias como de proteínas, assim como sua preferência habitual por alimentos com baixo conteúdo nutricional ${ }^{4}$ e muitas vezes ingestão em frequência insuficiente ${ }^{4,5}$. As infecções virais, bacterianas e parasitárias repetidas, as fomes causadas por guerras, secas ou outros desastres naturais e as práticas inadequadas de cuidado infantil, tais como administração de alimentos muito diluídos e/ou não higienicamente preparados, também podem ser citados como importantes fatores que levam à desnutrição $0^{4}$.

A desnutrição pode causar sequelas e/ ou comprometimento em algumas áreas do desenvolvimento neuropsicomotor, prejudicando, por exemplo, o funcionamento intelectual ${ }^{6,7,8}$. Foram encontradas, por meio de exames de neuroimagem, alterações no sistema nervoso central de crianças desnutridas ${ }^{9}$, permanecendo ainda questionamentos a respeito das consequências do efeito deletério causado pela desnutrição no sistema nervoso em desenvolvimento, sendo encontrados poucos estudos recentes que avaliaram essa condição $0^{10}$.

Mansur e Neto ${ }^{7}$ destacam que é provável que a criança não atinja seu verdadeiro potencial genético caso tenha privação de alimentação adequada durante o período de zero a dois 
anos de idade. Alguns estudos ${ }^{11,12}$ indicam que áreas e funções do desenvolvimento afetadas pela desnutrição podem não ser completamente recuperadas, mesmo após a melhora nutricional.

Apesar de na literatura serem encontrados estudos que investigam a relação entre a desnutrição e alterações no desenvolvimento neuropsicosensoriomotor, há pouco enfoque nas habilidades motoras de lactentes. Devido à incidência ainda considerável da desnutrição infantil no Brasil e à escassa literatura sobre sua relação com o desenvolvimento motor, evidencia-se a importância da realização de novas pesquisas que investiguem essa condição.

Desta forma, o objetivo é verificar a associação entre o DMG e o estado nutricional.

\section{MÉTODO}

Foi realizado estudo transversal, casocontrole, pareado, com lactentes com e sem desnutrição, no qual foi avaliado o desenvolvimento motor grosso entre seis e dezoito meses de idade.

De maio a novembro de 2009 foi composta uma amostra conveniência de lactentes com idades entre seis e dezoito meses, de ambos os sexos, usuários de três Unidades Básicas de Saúde da cidade de Juiz de Fora (MG), com e sem desnutrição. Foram excluídos do estudo os nascidos com idade gestacional inferior a 37 semanas, com baixo peso ao nascimento (menor que $2500 \mathrm{~g}$ ), com Índice Apgar inferior a 8 no primeiro e/ ou quinto minutos de vida ou, ainda, que apresentavam comprometimento neurológico de origem central ou periférica, malformações congênitas, síndromes, alterações sensoriais, ortopédicas, cardiorrespiratórias ou outras alterações que poderiam comprometer o desenvolvimento ou a movimentação do lactente.
Compuseram o grupo de estudo, lactentes com desnutrição, cadastrados no Serviço de Atenção ao Desnutrido (SAD) da Secretaria Municipal de Saúde de Juiz de Fora, MG. O grupo controle foi composto por lactentes sem desnutrição, pareados com os participantes do grupo de estudo (desnutridos) quanto à idade, sexo e nível socioeconômico, sendo recrutado através de busca ativa nas Unidades de Saúde envolvidas. Os pais assinaram o Termo de Consentimento Livre e Esclarecido para participação da pesquisa. O projeto foi autorizado pela Secretaria Municipal de Saúde e aprovado pelo Comitê de Ética em Pesquisa com Seres Humanos da Universidade Federal de Juiz de Fora - UFJF, sob parecer no $481 / 2008$.

Nos meses de março e abril de 2009, foi realizado estudo piloto nas Unidades de Saúde participantes e foram encontrados 34 lactentes cadastrados no SAD dentro da faixa etária de interesse. Considerando o pequeno universo de potenciais participantes, o fato de alguns poderem não ser localizados e ainda de outros se recusarem a participar, todos que no período de recrutamento contemplaram adequadamente os critérios de inclusão e exclusão foram convidados a fazer parte do grupo de estudo. Ao todo, 25 famílias de lactentes com desnutrição foram consultadas, destas, 20 aceitaram participar e 13 compareceram para a avaliação, sendo realizadas várias tentativas, sem sucesso, de agendamento dos outros sete casos. Apesar do ressarcimento das eventuais despesas com transporte oferecido aos participantes, alguns pais/responsáveis alegaram outros impedimentos para o não comparecimento nas datas marcadas para as avaliações como: doença do lactente (gripe, febre, infecções ou alterações gastrointestinais), mau tempo (chuva) e acontecimento de imprevistos com os pais/responsáveis.

O grupo controle foi sendo composto gradativamente, após a realização da avaliação de cada participante do grupo de estudo, segundo pareamento acima descrito. 
Para avaliação do desenvolvimento motor grosso foi utilizada a Alberta Infant Motor Scale $^{14}$ (AIMS), uma escala observacional que requer manuseio mínimo do lactente, podendo ser aplicada em 10 a 20 minutos. Pode ser utilizada em lactentes entre uma semana e 18 meses de vida e apresenta características psicométricas adequadas para identificar atrasos ou desvios no desenvolvimento motor grosso ${ }^{15}$. Sua aplicação e interpretação foi feita de acordo com o recomendado no manual da AIMS $^{14}$ e em artigos publicados sobre o seu uso ${ }^{15,16,17}$.

Para o presente estudo os lactentes foram avaliados por quatro membros de uma equipe previamente treinada (ICC: 0.99 ), nas Unidades de Saúde onde realizavam habitualmente o seu acompanhamento, próximo às datas de completarem $6,7,8,9,10,11,12,13$, $14,15,16,17$ ou 18 meses, permitindo uma variação de sete dias para mais ou para menos. Para análise, foram utilizados o Escore Bruto e o Índice Percentil de cada lactente, sendo este último transformado em uma variável categórica considerando como alterado o desempenho que estivesse abaixo do percentil 5 da curva de desenvolvimento normativa da escala. Apesar de ter sido inicialmente programado, devido às características físicas decorrentes da desnutrição, não foi possível realizar o cegamento da coleta de dados.

Foram ainda coletados dados referentes ao tempo de aleitamento (em meses), tempo de desnutrição (em meses), renda per capita (em reais), escolaridade dos pais e das mães (em anos de estudo cursados com aproveitamento), e nível socioeconômico conforme a Classificação Econômica Brasil ${ }^{18}$ (critério ABEP - Associação Brasileira de Empresas de Pesquisa). O critério da ABEP considera aspectos relativos ao número de cômodos e utensílios domésticos, o nível de escolaridade do chefe de família, estimando o poder de compra das pessoas e famílias urbanas, sendo sua divisão definida em classes econômicas. É muito utilizada para a caracterização da popu- lação brasileira e indica que 66\% desta pertencem às classes econômicas $\mathrm{C}$ e $\mathrm{D}$, em uma escala que varia, em ordem decrescente, de A a $\mathrm{E}$ (com algumas subdivisões).

Todos os dados foram registrados no cartão individual dos participantes e posteriormente arquivados em banco de dados no programa SPSS 14.0. Como os dados não satisfizeram os critérios de hipótese de normalidade, para a análise estatística foram utilizados testes não paramétricos (Mann-Whitney e Exato de Fisher), considerando um nível de significância $\alpha=0,05$. O teste de MannWhitney foi aplicado para comparação entre os grupos do Escore Bruto obtido na Alberta Infant Motor Scale (AIMS), da renda per capita e do tempo de estudo dos pais (variáveis numéricas). Para comparação da classificação segundo o Índice Percentil na AIMS (variável categórica) foi utilizado o teste Exato de Fisher.

\section{RESULTADOS}

A amostra ficou composta por 26 participantes, em sua maioria acima de um ano (10/ 76,95\%), sendo 13 lactentes no grupo de estudo e 13 no grupo controle, e suas características estão descritas na Tabela 1. As variáveis idade, sexo e nível socioeconômico (ABEP) foram pareadas, não havendo, portanto, diferença entre os grupos. As demais variáveis contínuas que não foram controladas (renda per capita e tempo de estudo dos pais) também não apresentaram diferenças estatisticamente significantes segundo o teste de Mann-Whitney. O tempo de aleitamento foi analisado como uma variável categórica, não sendo possível verificar a semelhança ou não entre os grupos, devido ao pequeno número de participantes em cada sub-categoria, mas a distribuição indicou semelhança. A idade dos participantes variou entre oito e 18 meses, o tempo de desnutrição entre um e 12 meses, a renda per capta variou de $\mathrm{R} \$ 83,33$ a $\mathrm{R} \$$ 
416,67, a escolaridade da mãe entre 0 e 11 anos e a escolaridade do pai entre 4 e 15 anos. Por desconhecimento do informante, não foi possível saber a escolaridade do pai de 6 participantes (três do grupo de estudo e três do grupo controle).

Tabela 1: Características dos participantes por grupo (estudo e controle) quanto ao sexo, classificação socioeconômica (ABEP), tempo de aleitamento (número absoluto), idade, escolaridade dos pais, tempo de desnutrição e renda per capita (média e desvio padrão).

\begin{tabular}{|c|c|c|}
\hline Variáveis & Grupo de estudo & Grupo controle \\
\hline \multicolumn{3}{|l|}{ Sexo } \\
\hline Masculino & 7 & 7 \\
\hline Feminino & 6 & 6 \\
\hline \multicolumn{3}{|l|}{ ABEP } \\
\hline B2 & 2 & 2 \\
\hline $\mathrm{C} 1$ & 5 & 5 \\
\hline $\mathrm{C} 2$ & 4 & 4 \\
\hline $\mathrm{D}$ & 2 & 2 \\
\hline \multicolumn{3}{|l|}{ Tempo de aleitamento } \\
\hline$<=2$ meses & 2 & 3 \\
\hline$>2 \mathrm{e}<=4$ meses & 0 & 1 \\
\hline$>4$ meses & 11 & 9 \\
\hline Idade (meses) & $14,54 \pm 3,23$ & $14,54 \pm 3,23$ \\
\hline Escolaridade do pai (anos) & $7,60 \pm 3,20$ & $8,60 \pm 2,55$ \\
\hline Escolaridade da mãe (anos) & $8,77 \pm 2,01$ & $8,77 \pm 3,22$ \\
\hline Tempo de desnutrição (meses) & $5,85 \pm 3,72$ & 0 \\
\hline Renda per capita (reais) & $147,42 \pm 37,53$ & $203,76 \pm 93,30$ \\
\hline
\end{tabular}

O desempenho dos participantes na Alberta Infant Motor Scale (AIMS) está descrito nas tabelas 2 e 3. Considerando tanto o Escore Bruto quanto o Índice Percentil, foi encontrada diferença estatisticamente significante entre os grupos ( $\mathrm{p}=0,023 \mathrm{e}$ $\mathrm{p}=0,002$, respectivamente), tendo sido observado desempenho alterado (abaixo do percentil 5) em 10 (76,9\%) lactentes do grupo de estudo e em dois $(15,4 \%)$ do grupo controle. Diferentemente do grupo controle, foi observada grande dispersão no escore bruto dos participantes do grupo de estudo (alto valor de desvio-padrão e grande diferença entre os valores mínimo e máximo).

\section{DISCUSSÃO}

O presente estudo acrescenta importantes considerações em relação à associação da desnutrição com o pobre desempenho motor de lactentes entre seis e 18 meses de idade: os participantes do grupo de estudo (com desnutrição) demonstraram desempenho na Alberta Infant Motor Scale (AIMS) significativamente inferior ao do grupo controle, considerando tanto o Escore Bruto quanto o Índice Percentil. Os participantes que obtiveram Índice Percentil abaixo de cinco foram considerados com desempenho alterado, sendo que a grande maioria do grupo de estudo (10/ 76,9\%) recebeu esta classificação. 
Tabela 2: Desempenho dos participantes segundo o escore bruto obtido na AIMS e p-valor (Teste Mann-Whitney).

\begin{tabular}{lrrrrrc}
\hline Escore Bruto & $\mathbf{n}$ & Média \pm DP & Mín. & Median. & Máx & p-valor \\
\hline Grupo Estudo & 13 & $42,62 \pm 14,99$ & 16,00 & 46,00 & 58,00 & \multirow{2}{*}{0,023} \\
Grupo Controle & 13 & $53,92 \pm 6,97$ & 35,00 & 57,00 & 58,00 & \\
\hline
\end{tabular}

$\mathrm{n}=$ número de participantes; $\mathrm{DP}=$ desvio-padrão

Tabela 3: Classificação do desempenho dos participantes segundo o índice percentil obtido na AIMS ( $\mathrm{P}>=5$ ou $\mathrm{P}<5$ (alterado)) e $p$-valor (Teste Exato de Fisher).

\begin{tabular}{lrrc}
\hline Índice Percentil & $\mathbf{P}>=\mathbf{5}(\mathbf{n} / \mathbf{\%})$ & $\mathbf{P}<\mathbf{5}(\mathbf{n} / \mathbf{\%})$ & $\boldsymbol{p}$-valor \\
\hline Grupo Estudo & $3 / 23,1 \%$ & $10 / 76,9 \%$ & 0,002 \\
Grupo Controle & $11 / 84,6 \%$ & $2 / 15,4 \%$ & \\
\hline
\end{tabular}

$\mathrm{n}=$ número de participantes; $\mathrm{P}=$ percentil

Não foram encontrados na literatura estudos que utilizaram a AIMS para avaliar o desempenho motor de lactentes desnutridos, mas alguns avaliaram tal desempenho utilizando outras escalas ${ }^{13,20,21,22}$.

Corroborando os achados do presente estudo, Drewett et $\mathrm{al}^{20}$ e Hamadani et $\mathrm{al}^{21}$ avaliaram o desenvolvimento motor de desnutridos e não desnutridos por meio da Escala Motora de Bayley e encontraram escores significativamente inferiores no grupo de desnutridos. Gardner et $\mathrm{al}^{22}$ também encontraram déficits no desenvolvimento locomotor de lactentes de um ano com desnutrição, por meio da avaliação com a Griffith's Mental Development Scales.

Ao contrário do encontrado no presente estudo e nos citados no parágrafo anterior, Mansur e Neto ${ }^{7}$ verificaram, por meio da escala de Desenvolvimento Psicomotor da Primeira Infância de Brunet e Lézine, em lactentes desnutridos de sete a 24 meses (média de 16,41 meses), que somente as áreas de sociabilidade e linguagem obtiveram índices com valores abaixo da média para a idade cronológica. É importante ressaltar que no referido estudo não houve grupo controle, sendo o desempenho dos participantes comparado apenas com os esco- res normativos da escala. Além disto, a escala utilizada poderia ser outro fator que explicaria esta contradição, uma vez que, diferentemente da AIMS, a escala de Desenvolvimento Psicomotor da Primeira Infância de Brunet e Lézine também considera aspectos do desenvolvimento motor fino.

Nesse mesmo sentido, Almeida ${ }^{13}$ afirma não haver diferença estatisticamente significante entre os desempenhos médios do desenvolvimento psicomotor (PDI), utilizando a Escala Motora de Bayley, entre amostras de crianças com e sem carência nutricional. Chama atenção o fato de que, no referido estudo, não houve pareamento das amostras (idade, sexo), a faixa etária foi mais ampla (4 a 42 meses), não houve critério de exclusão (sendo incluídos, tanto no grupo de estudo como no controle, lactentes nascidos com baixo peso, prematuridade e outros fatores de risco) e o grupo de estudo envolveu crianças com carência nutricional decorrente tanto do baixo peso como de anemia ferropriva (não tendo sido descrito o número de participantes com cada uma destas condições). Todos esses fatores podem ter interferido nos achados, sendo que a própria autora cita este último como limitação do estudo. Ainda, no estudo de Almeida ${ }^{13}$, as médias 
de idade dos participantes foram mais altas (20,93 meses no grupo de estudo e 28,5 meses no grupo controle), com diferença estatisticamente significante entre os grupos, o que também pode ter influenciado os achados.

Drewett et $\mathrm{al}^{20}$ encontraram diferenças significativas entre os escores obtidos na Escala Bayley de lactentes com dois anos de idade que adquiriram desnutrição antes e após seis meses de idade. Desta forma, pode-se levantar a hipótese de que a desnutrição afeta o desenvolvimento de forma diferente em cada fase ou idade. Esta condição pode se instalar em um momento em que algumas aquisições motoras já tenham sido adquiridas e, portanto, não sendo tão afetadas.

Os mecanismos envolvidos com a desnutrição durante o período do desenvolvimento crítico do cérebro, segundo BenítezBribiesca et $\mathrm{al}^{23}$, podem levar a alterações como o atraso no crescimento do cérebro, anomalias morfológicas e funcionais dos neurônios, principalmente na sinapse dendrítica o que causa a pobre integração neuronal, podendo explicar os déficits neuropsicológicos que ocorrem nos desnutridos. Para Taner et $\mathrm{al}^{24}$, a criança que tem a fome, que não é saciada, pode perder a motivação para explorar o ambiente o que, adicionalmente à questão metabólica/fisiológica, levaria ao atraso para a aquisição de certas habilidades cognitivas.

Corroborando Taner et al ${ }^{24}$ e Pollitt ${ }^{25}$ sugerem mecanismos por meio dos quais a desnutrição leva à redução do comportamento exploratório. O pequeno tamanho do corpo, as proporções antropométricas, a fraqueza muscular e a maturação poderiam impor limites imediatos na aquisição das habilidades motoras o que interfere na exploração e, consequentemente na atividade física. Tais características poderiam induzir os pais a tratarem a criança como menos competente do que ela realmente poderia ser criando um círculo vicioso. Esta máxima vai ao encontro do que sugerem Aboud e Alemu ${ }^{26}$ que descrevem que as mães dos desnutridos interagem menos com eles ocasionando possivelmente um atraso no desenvolvimento mental.

Nesse sentido, o estudo de Alvarez et al ${ }^{19}$ encontrou diferenças de desempenho entre os desnutridos que pareciam associadas à qualidade e/ou quantidade da estimulação ambiental recebida pelos mesmos. Os lactentes que receberam menos estimulação ambiental apresentaram desempenho motor, segundo a Escala Bayley, significativamente inferior ao dos lactentes com maior estimulação.

Considerando os possíveis fatores indicados na literatura como associados ao desenvolvimento nos primeiros anos de vida (escolaridade dos pais ${ }^{13}$, nível socioecônomico ${ }^{27}$, início da desnutrição ${ }^{20}$, tempo de aleitamento ${ }^{27}$ ), no presente estudo foi feito o pareamento das amostras e verificada a semelhança ou não das variáveis contínuas que não foram pareadas, sendo que, as diferenças encontradas no desempenho motor dos grupos parecem estar associadas ao estado nutricional e não a estes outros fatores.

Chama a atenção o fato de dois lactentes do grupo controle $(15,4 \%)$ terem apresentado desempenho alterado. Por não ser objetivo do presente estudo e devido ao desenho empregado, pode-se apenas levantar algumas hipóteses sobre esse achado. A primeira seria a possibilidade de estes dois lactentes terem sido avaliados em um momento de evolução mais lenta do seu desenvolvimento (momento Platô), devido à variabilidade intra-individual que existe mesmo em lactentes típicos ${ }^{16}$, ou em um dia em que as condições não eram as mais favoráveis (p. ex.: sono noturno agitado ou em menor quantidade). Além disso, possíveis variações nos estímulos ambientais e na interação com a mãe $e^{19}$, fatores que não foram controlados neste estudo, podem ter influenciado.

Os presentes achados contribuem para reafirmar a importância de uma abordagem integral ao desnutrido que vá além da recuperação da condição nutricional, como o preconizado nos documentos da Secretaria de Esta- 
do da Saúde de Minas Gerais ${ }^{5}$ e do $\mathrm{UNICEF}^{28}$, incluindo a vigilância do desenvolvimento motor e, quando necessário, a realização de medidas que visem melhorar essa condição.

É importante salientar que, segundo critérios do programa, os lactentes são cadastrados no Serviço de Atenção ao Desnutrido (SAD) da Secretaria Municipal de Saúde de Juiz de Fora após os seis meses de idade, mas parece haver uma sub-notificação no primeiro ano de vida ou esta condição se instala mais tardiamente na região onde foi realizada a pesquisa, pois o grupo de estudo foi composto em sua maioria por lactentes acima de um ano.

\section{REFERÊNCIAS}

1. Monte CMG. Desnutrição: um desafio secular à nutrição infantil. J Pediatr. Rio J. 2000; 76 Supl 3:S285-9.

2. ACC/SCN. Nutrition throughout life. 4th Report on the world nutrition situation. Geneva: ACC/SCN./World Health Organization; 2000 .

3. Instituto Brasileiro de Geografia e Estatística. Pesquisa de Orçamentos Familiares 2002-2003: antropometria e análise do estado nutricional de crianças e adolescentes no Brasil. Rio de Janeiro: Instituto Brasileiro de Geografia e Estatística; 2006. [acesso em 15 nov 2009]. Disponível em: http://www.ibge.gov.br/home/estatistica/ populacao/condicaodevida/pof/2002analise/default.shtm

4. WHO. Complementary feeding of young children in developing countries. Geneva: World Health Organization;1998. [acesso em 23 nov 2008]. Disponível em: http://whqlibdoc.who.int/hq/1998/ WHO_NUT_98.1.pdf
Concluindo, lactentes com desnutrição de seis a 18 meses de idade apresentaram desenvolvimento motor significativamente inferior aos seus pares, com bom estado nutricional, indicando que a desnutrição parece estar negativamente associada ao desenvolvimento motor grosso nesta faixa etária. Estudos adicionais são necessários para confirmar ou não os achados e para investigar outros aspectos que podem estar associados a esta condição como a contribuição do tempo de desnutrição, a fase do desenvolvimento em que a mesma aparece e os estímulos ambientais oferecidos.

5. Alves CRL, Alvim CG, Junqueira HS, Goulart LMHF, Dias LS, Magalhães MEN et al. Atenção à Saúde da Criança. Secretaria de Estado de Saúde de Minas Gerais. $1^{\mathrm{a}}$ ed. Belo Horizionte: SAS/DNAS; 2005. p. 85.

6. Plaza B, Brito NI, Torrejón HP, Gloor VC, Medina JMJ, Díaz TT et al. Algunas consideraciones sobre el impacto de la desnutricion en el desarrollo cerebral, inteligencia y rendimiento escolar. ALAN 2001; 51(1): 64-71.

7. Mansur SS, Neto FR. Desenvolvimento neuropsicomotor de lactentes desnutridos. Rev Bras Fisioter. 2006;10(2):185-91.

8. Meio MDB, Lopes CS, Morsch DS. Fatores prognósticos para o desenvolvimento cognitivo de prematuros de muito baixo peso. Rev Saúde Pública. 2003; 37(3): 311-8.

9. Marcondes E. Desidratação e desnutrição em pediatria. São Paulo: Sarvier; 1971.

10. Nunes ML. Desnutrição e desenvolvimento neuropsicomotor. J Pediatr (Rio J). 2001;77(3):159-60. 
11. McKay H, Sinisterra L, McKay A, Gómez $\mathrm{H}$, Lloreda P. Improving cognitive ability in chronically deprived children. Science (Wash.). 1978; 200: 270-8.

12. Galler JR, Ramsey F, Solimano G, Kuscharski LT, Harrison R. The influence of early malnutrition on subsequent behavioral development. iv. Soft neurologic signs. Pediatr Res. 1984;18(9):826-32.

13. Almeida PS. Avaliação do desenvolvimento mental e psicomotor de crianças de creche com carências nutricionais [dissertação]. Ribeirão Preto: Universidade de São Paulo; 2004.

14. Darrah J, Piper M, Watt MI. Assessment of gross motor skills of at risk infants: predictive validity of the Alberta Infant Motor Scale. Dev Med Child Neurol.. 1998;40:485-91.

15. Piper MC, Darrah J. Motor Assessment of Developing Infant. W B Saunders 1994.

16. Darrah J, Redfern L, Maguire TO, Beaulne $\mathrm{P}$, Watt J. Intra-individual stability of rate of gross motor development in full-term infants. Early Hum Dev. 1998;52:169-79.

17. Campbell SK, Kolobe THA, Wright BD, Linacre JM. Validity of the test of infant motor performance for prediction of 6-9and 12-month scores on the Alberta Infant Motor Scale. Dev Med Child Neurol. 2002;44:263-72.

18. Associação Brasileira de Empresas de Pesquisa (ABEP): Critério de classificação econômica Brasil 2008. [acesso em 20 jun 2008]. Disponível em: http:// www.abep.org

19. Alvarez MDLL, Concha X, Elordi M, Lamilla C, Ramos C, Perez P. Desnutrición infantil, coeficiente de desarrollo y su relación con el medio ambiente: un estudio piloto. Rev Saúde Pública. 1991; 25(4): 282-8.
20. Drewett R, Wolke D, Azefa M, Kaba M, Tessema F. Malnutrition and mental development: is there a sensitive period? A nested case-control study. J Child Psychol Psychiat. 2001;42(2):181-7.

21. Hamadani JD, Huda SN, Khatun F, Grantham-McGregor SM. Psychosocial stimulation improves the development of undernourished children in rural Bangladesh. J Nutr. 2006; 136: 2645-52.

22. Gardner JMM, Grantham-McGregor SM, Chang SM. Activity and behavioral development in stunted and nonstunted children and response to nutritional supplementation. Child Dev.1995;66:1785-97.

23. Benítez-Bribiesca L, Rosa-Alvarez IDL, Mansilla-Olivares A. Dendritic spine pathology in infants with severe proteincalorie malnutrition. Pediatrics. 1999; 104(2): 1-6.

24. Taner EM, Finn-Stevenson M. Nutrition and brain development: social policy implications. Am J Orthopsychiatr. 2002; 72(2): 182-93.

25. Pollitt E. A developmental view of the undernourished child: background and purpose of the study in Pangalengan, Indonesia. Eur J Clin Nutr. 2000;54 Supl 2, S2-10.

26. Aboud FE, Alemu T. Nutrition, maternal responsiveness and mental development of ethiopian children. Soc Sci Med (1982). 1995;41(5):725-32.

27. Halpern R, Giugliani ERJ, Victoria CG, Barros FC, Horta BL. Fatores de risco para a suspeita de atraso no desenvolvimento neuropsicomotor aos 12 meses de vida. J Pediatr (Rio J). 2000;76:421-8.

28. UNICEF. The state of the world's children 1998. Oxford: United Nations Children’s Fund/Oxford University Press;1997.

Recebido em: 06/abr./10 Modificado em: 16/ago./10 Aceito em: 09/set./10 